\title{
AN EXTREMAL PROBLEM IN HYPERGRAPH THEORY (II)
}

\author{
H. L. ABBOTT, D. HANSON and A. C. LIU
}

(Received 22 April 1980)

Communicated by W. D. Wallis

\begin{abstract}
Let $t, m>2$ and $p>2$ be positive integers and denote by $N(t, m, p)$ the largest integer for which there exists a $t$-uniform hypergraph with $N$ (not necessarily distinct) edges and having no independent set of edges of size $m$ and no vertex of degree exceeding $p$. In this paper we complete the determination of $N(t, m, 3)$ and obtain some new bounds on $N(t, 2, p)$.
\end{abstract}

1980 Mathematics subject classification (Amer. Math. Soc.): 05 C 65.

\section{Introduction}

We continue in this paper our study of the following combinatorial problem which we investigated in [1], [2] and [3]. Let $t, m>2$ and $p>2$ be positive integers and denote by $N=N(t, m, p)$ the largest integer for which there exists a $t$-uniform hypergraph with $N$ (not necessarily distinct) edges and having no independent set of edges of size $m$ and no vertex of degree exceeding $p$. Such a graph will be called a $(t, m, p)$-graph.

The problem of evaluating $N(t, m, p)$ for all values of the parameters seems to be very difficult. In our earlier work we established some upper and lower bounds and obtained exact values of $N(t, m, p)$ for various infinite classes of values of $t, m$ and $p$. In this paper, we obtain further exact values and some improvement on bounds.

In [1], we proved that

$$
\begin{array}{ll}
N(t, m, 3)=(2 t+1)(m-1) & \text { if } t \equiv 0,1(\bmod 3) \\
(2 t-1)(m-1)<N(t, m, 3) \leqslant(2 t+1)(m-1) & \text { if } t \equiv 2(\bmod 3) .
\end{array}
$$

Copyright Australian Mathematical Society 1981 
We complete the determination of $N(t, m, 3)$ by proving

TheOREM A. $N(t, m, 3)=2 t(m-1)$ if $t \equiv 2(\bmod 3)$.

In [1], we also proved that

$$
N(t, 2, p) \leqslant t p-t+1 \text {. }
$$

We pointed out that equality holds in (2) whenever there exists a block design $B(b, \nu, r, k, \lambda)$ with $r=t, k=p$ and $\lambda=1$. In [2], we showed that if there exists a projective plane of order $t-1$ and if $p \equiv 0(\bmod t)$, then

$$
N(t, 2, p) \geqslant p\left(t^{2}-t+1\right) / t .
$$

Consequently, if $t$ is large and $p$ is large compared to it, then the bound given by (2) is asymptotically correct.

We prove in this paper that if $t$ is considered fixed, the bound given by (2) can be significantly improved for large $p$. It will be convenient to formulate our results in terms of

$$
\beta_{t}=\lim _{p \rightarrow \infty} \frac{N(t, 2, p)}{p} .
$$

It follows from (2) that $\beta_{t} \leqslant t$. We shall prove the following result:

THEOREM B. For all $t \geqslant 2$,

$$
\beta_{t} \leqslant t-1+\max _{n}\left\{\frac{n\left(t^{2}-2 t\right)-t^{4}+4 t^{3}-6 t^{2}+4 t}{n^{2}-n(2 t+1)+t^{3}-2 t^{2}+3 t}\right\}
$$

where the maximum is taken over all $n \geq t^{2}-t+1$.

In what follows we denote the degree of a vertex $x$ of a graph $\mathscr{F}$ by $d(x)$ and $d_{\mathcal{G}}(x)$ will denote the degree of $x$ in the subgraph $\mathcal{G}$ of $\mathscr{F}$.

\section{Proof of Theorem A}

We use induction on $m$. Consider first the case $m=2$. We have from (1) that $2 t-1 \leqslant N(t, 2,3) \leqslant 2 t+1$. That $N(t, 2,3)>2 t$ is shown by the following explicit construction: Take a block design $B(b, \nu, r, k, \lambda)$ with parameters $\nu=2 t$ $-1, b=((t-1)(2 t-1)) / 3, r=t-1, k=3, \lambda=1$, that is a Steiner triple system. The condition $t \equiv 2(\bmod 3)$ is sufficient to ensure that such a design exists. Let the elements be $v_{1}, v_{2}, \ldots, v_{v}$ and let the blocks be $B_{1}, B_{2}, \ldots, B_{b}$. Let $A$ be the incidence matrix of the design so that $A=\left[a_{i j}\right]$ where $a_{i j}=1$ if $v_{j} \in B_{i}$ and 0 otherwise. Let $\mathcal{G}$ be the family of sets whose incidence matrix is 
$A^{T}$, the transpose of $A$. The members of $\mathcal{G}$ may be thought of as the edges of a $(t-1)$-uniform hypergraph. Note that each vertex has degree three and each pair of edges intersect. Let $G_{1}, G_{2}, \ldots, G_{2 t-1}$ be the edges of $\mathcal{G}$. Let $E=$ $\left\{u_{1}, u_{2}, \ldots, u_{t}\right\}$ be a set which is disjoint from $\cup \mathcal{G}$. For $i=1,2, \ldots, t$ let $F_{2 i-1}=G_{2 i-1} \cup\left\{u_{i}\right\}$ and for $i=1,2, \ldots, t-1$ let $F_{2 i}=G_{2 i} \cup\left\{u_{i}\right\}$. Let $\mathscr{F}$ be the $t$-uniform hypergraph whose edges are $E, F_{1}, F_{2}, \ldots, F_{2 t-1}$. Then it is a simple matter to check that $\mathscr{F}$ has all of the properties needed to establish that $N(t, 2,3)>2 t$.

We now have to rule out the possibility that $N(t, 2,3)=2 t+1$. Suppose $N(t, 2,3)=2 t+1$ and let $\mathscr{F}$ be a $(t, 2,3)$ graph. Not all vertices of $\mathscr{F}$ have degree three since this would give $t N \equiv 0(\bmod 3)$. Thus there is a vertex $v$ which has degree at most 2 . Then if $v \in F$ we have

$$
|\mathcal{F}|<d(v)+\sum_{\substack{u \in F \\ u \neq v}}(d(u)-1) \leqslant 2 t
$$

a contradiction. Thus $N(t, 2,3)=2 t$.

It turns out that in order to make the induction argument go through we need a fairly strong induction hypothesis. We record first certain properties of the $(t, 2,3)$ graphs which we shall need to make use of later.

(a) A $(t, 2,3)$ graph has no vertex of degree one.

(b) A $(t, 2,3)$ graph has at least one vertex of degree two, since otherwise we would have $t N=2 t^{2} \equiv 0(\bmod 3)$.

(c) If a $(t, 2,3)$ graph has two vertices of degree two, they do not appear in the same edge.

(d) A $(t, 2,3)$ graph does not have $t$ or more vertices of degree two. (It is clear, by (c) that there cannot be more than $t$ vertices of degree two. If there were $t$ such vertices, each edge would have to contain exactly one vertex of degree two and all other vertices would be of degree three, so that if $s$ is the number of vertices of degree three we have $2 t+3 s=t N=2 t^{2}$, but this implies $t \equiv$ $1(\bmod 3)$.)

Now let $m>2$ and take, as the induction hypothesis, the following statement: for $2<k<m, N(t, k, 3)=2 t(k-1)$ and component of a $(t, k, 3)$ graph is either a $(t, 2,3)$ graph or a $(t, l, 3)$ graph, $l<k$ in which each vertex is of degree three.

Let $\mathscr{F}$ be a $(t, m, 3)$ graph. If $\mathscr{F}$ is not connected, the desired conclusion follows immediately from the induction hypothesis. Hence we suppose that $\mathscr{F}$ is connected. We need to show that $|\mathscr{F}|=2 t(m-1)$ and that all vertices of $\mathscr{F}$ have degree 3 . We may suppose that $n(>2)$ is the least integer for which a connected $(t, m, 3)$ graph exists. 
We note that if $v$ is a vertex of $\mathscr{F}$ and if $v \in E$ then

$$
|\mathscr{F}| \leqslant d(v)+\sum_{\substack{u \in E \\ u \neq v}}(d(u)-1)+\sum_{\substack{F \in \mathscr{F} \\ F \cap E=\varnothing}} 1
$$

It is an immediate consequence of (5) and the induction hypothesis that $\mathscr{F}$ has no vertex of degree one. We therefore need to consider two cases.

Case 1. F has a vertex of degree two. Let $v$ be a vertex of degree two and let $v \in E$. It follows from (5) that

$$
|\mathscr{F}| \leqslant 2+2(t-1)+N(t, m-1,3)=2 t(m-1)
$$

so that $|\mathscr{F}|=2 t(m-1)$. Let $\mathscr{Q}=\{F: F \in \mathscr{F}, F \cap E \neq \varnothing\}$ and let $\mathscr{B}=$ $\{F: F \in \mathscr{F}, F \cap E=\varnothing\}$. It is clear that $|\mathscr{Q}|<2 t$. If $|\mathbb{Q}|<2 t$, then $|\mathscr{B}|>$ $2 t(m-2)$, so that there is an independent set of edges in $\mathscr{B}$ of size $m-1$. This set, together with $E$, gives an independent set of size $m$ in $\mathscr{F}$. Thus $|\mathbb{Q}|=2 t$, and $|\mathscr{B}|=2 t(m-2)=N(t, m-1,3)$. It follows that $\mathscr{B}$ is a $(t, m-1,3)$ graph. By the induction hypothesis and the minimality of $m, \mathscr{B}$ has components $H_{1}$, $H_{2}, \ldots, H_{m-2}$ which are $(t, 2,3)$ graphs. Since $\mathscr{F}$ is connected, there exists a vertex $x$ such that $x \in \cup \mathscr{Q}$ and $x \in \cup \mathscr{B}$. The structure of $\mathscr{B}$ and (a) imply that $x$ must appear in two members of $\mathscr{B}$ and in only one member of $\mathscr{C}$. By (a), $\mathbb{Q}$ is not a $(t, 2,3)$ graph. Hence there are two sets $F_{1}, F_{2} \in \mathbb{Q}$ which are disjoint. There cannot exist in each $H_{i}$ a set which is disjoint from $F_{1} \cup F_{2}$ since this would clearly yield an independent set in $\mathscr{F}$ of size $m$. Hence for some $j, 1 \leqslant j \leqslant m-2$, every member of $H_{j}$ intersects $F_{1} \cup F_{2}$, and by (c), in exactly one place. This implies that $H_{j}$ contains $t$ vertices $x$ such that $d_{H_{j}}(x)=2$, contrary to (d).

Case 2. All vertices of $\mathscr{F}$ have degree three. It follows from (5) and the induction hypothesis that $|\mathscr{F}| \leqslant 2 t+1+N(t, m-1,3)=2 t(m-1)+1$. Thus all that remains is to rule out the possibility $|\mathscr{F}|=2 t(m-1)+1$. Let $E$ be an edge of $\mathscr{F}$ and let $\mathscr{Q}=\{F: F \in \mathscr{F}, F \cap E \neq \varnothing\}$ and let $\mathscr{B}=\{F: F \in \mathscr{F}$, $F \cap E=\varnothing\}$. Then $|\mathscr{Q}| \leqslant 2 t+1$. If $|\mathscr{Q}| \leqslant 2 t$ we have $|\mathscr{B}|>N(t, m-1,3)$ so that $\mathscr{B}$ contains an independent set of size $m-1$ and this, with $E$, gives an independent set of size $m$ in $\mathscr{F}$. Hence we may suppose $|\mathscr{Q}|=2 t+1,|\mathscr{B}|=$ $2 t(m-2)$ and $\mathscr{B}$ is a $(t, m-1,3)$ graph. Since $\mathscr{F}$ is connected, not all vertices $x$ in $\cup \mathscr{B}$ satisfy $d(x)=3$. Thus, by the minimality of $m, \mathscr{B}$ has components $H_{1}, H_{2}, \ldots, H_{m-2}$ where each $H_{i}$ is a $(t, 2,3)$ graph. Now $|\mathfrak{Q}|>2 t=N(t, 2,3)$ implies that there are two members of $Q$, say $F_{1}$ and $F_{2}$, which are disjoint. The remainder of the argument now parallels that given in Case 1 . Thus $|\mathscr{F}|=$ $2 t(m-1)+1$ cannot occur. This completes the proof of the theorem. 
We do not know whether the $(t, m, 3)$ graphs for $m>3$ consist of $m-1$ components, although we suspect that this is the case. Such is not the case in general, however. For example, as was pointed out in $[3], N(3,3,4)=16$ and the following graph is a connected $(3,3,4)$ graph. The heavy edges have multiplicity two and all others have multiplicity one.

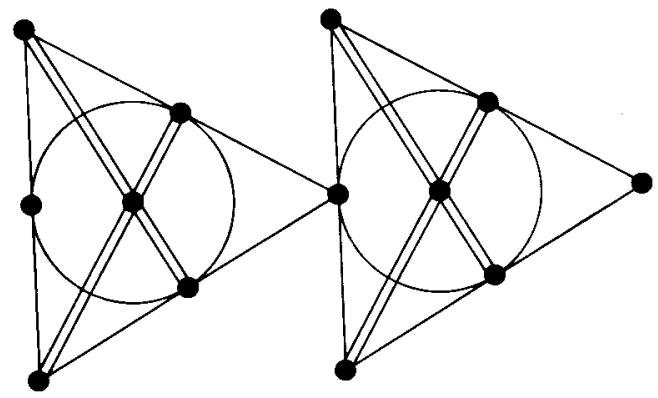

\section{Proof of Theorem B}

Let $h \geqslant 0$ be defined by

$$
N(t, 2, p)=t p-t+1-h
$$

and let $\mathscr{F}$ be an extremal graph; that is, $\mathscr{F}$ has $N=N(t, 2, p)$ edges, maximal degree $p$ and any two edges of $\mathscr{F}$ intersect.

Every edge of $\mathscr{F}$ has a vertex of degree $p$, since if there were an edge all vertices of which have degree less than $p$ the multiplicity of this edge could be increased. If follows from this observation that if there were fewer than $t$ vertices of degree $p$ then $N \leqslant(t-1) p$ and our theorem would be proved. Hence we may suppose there are at least $t$ vertices of degree $p$. Let $n$ denote the number of vertices of $\mathscr{F}$. We may suppose $n \geqslant t^{2}-t+1$ since otherwise we get $t N \leqslant p n$ $<p\left(t^{2}-t\right)$ so that $n<(t-1) p$. Let $v$ be a vertex of minimal degree. Then

$$
d(v)(n-t)+p t \leqslant t N
$$

and it follows from (6) and (7) that

$$
d(v) \leqslant \frac{t(t p-h-p-t+1)}{n-t} .
$$

Let $\mathcal{G}=\{F: F \in \mathscr{F}, v \in F\}$. Since $\sum_{x \neq v} d_{\mathscr{G}}(x)=(t-1) d(v)$ the average value of $d_{\mathcal{G}}(x)$ is $(t-1) d(x) /(n-1)$. It is clear that there exists $E \in \mathcal{G}$ which is "at least average" in the sense that

$$
\sum_{\substack{x \in E \\ x \neq v}} d_{\Theta}(x) \geqslant \frac{(t-1)^{2} d(v)}{n-1} .
$$


Thus

$$
\begin{aligned}
N & =|\mathcal{G}|+|\mathscr{F}-\mathcal{G}|=d(v)+\sum_{\substack{\mathcal{F} \notin+0 \\
F \notin \mathcal{E}}} 1 \\
& \leqslant d(v)+(t-1) p-\sum_{\substack{x \in E \\
x \neq v}} d_{\mathcal{G}}(x),
\end{aligned}
$$

and this, with (9), gives

$$
N \leqslant d(v)+(t-1) p-\frac{(t-1)^{2} d(v)}{n-1}
$$

and it follows from (6) and (10) that

$$
d(v)>\frac{(n-1)(p-h-t+1)}{n-t^{2}+2 t-2} .
$$

The maximum value of the right side of (10), subject to (8) and (11) occurs when (8) and (11) hold with equality. One finds, after some routine manipulations, that

$$
N \leqslant\left\{t-1+\frac{n\left(t^{2}-2 t\right)-t^{4}+4 t^{3}-6 t^{2}+4 t}{n^{2}-n(2 t+1)+t^{3}-2 t^{2}+3 t}\right\} p+a(n, t)
$$

where $a(n, t)$ depends only on $n$ and $t$. The theorem now follows immediately.

Since the right side of (4) is fairly complicated, the improvement over (2) may not be apparent. We illustrate for the case $t=4$. When $t=4$, it follows from (2) and (3) that $3.25 \leqslant \beta_{4} \leqslant 4$, while (4) gives

$$
\beta_{4} \leqslant 3+\max _{n>13}\left(\frac{8 n-80}{n^{2}-9 n+44}\right) .
$$

One finds that the maximum occurs at $n=17$ so that $\beta_{4}<149 / 45<3.312$.

\section{References}

[1] H. L. Abbott, D. Hanson and A. C. Liu, 'An extremal problem in graph theory', Quart. J. Math., Oxford Ser. 131, 121, 1980.

[2] H. L. Abott, M. Katchalski and A. C. Liu, 'An extremal problem in graph theory II', $J$. Austral. Math. Soc., (to appear).

[3] H. L. Abbott, M. Katchalski and A. C. Liu, 'An extremal problem in hypergraph theory', Discrete Mathematical Analysis and Combinatorial Computation, Conference proceedings, School of Computer Science, University of New Brunswick, Fredericton, (1980) pp. 74-82. 
Mathematics Department

University of Alberta

Edmonton, Alberta

Canada

Mathematics Department

University of Alberta

Edmonton, Alberta

Canada
Department of Mathematics

University of Regina

Regina

Canada S4S 0A2 